\title{
The psychological aspect of pain and symptom management in clinical practices: Scoping review
}

\author{
Suantak Demkhosei Vaiphei \\ ${ }^{1}$ Department of Psychology, Assam Down Town University, Guwahati, India
}

Received: 2020-11-01.

Accepted: 2021-10-03

This work is licensed under a Creative Commons Attribution 4.0 International License

J Clin Med Kaz 2021; 18(6):4-8

Corresponding author:

Suantak Demkhosei Vaiphei.

E-mail: gdsuantak@yahoo.com;

ORCID: 0000-0002-4134-2926

\begin{abstract}
The concept of pain differs from individual personal physical, emotional, social, and cognitive-behavior conditions. Pain in its nature of existence is a complex psychoneurotic syndrome that requires a multidisciplinary inclusive approach to deal with complicated disorders. The psychological issues are a central concern in dealing with pain syndrome, resulting in maximizing emotional outbursts and disabilities. The particular immediate context of occurrences hugely influences the perception of intensity over acute pain syndrome. The cognitive-behavioral psychotherapeutic is the one approach that effectively addresses the nature of total pain in the context where it occurs and the associated factors. The pain syndrome needs to be applied by understanding specific components associated with pain and through the optimal comprehensive assessment interventions at a multidisciplinary level. The psychopharmacology aspect of pain management in conjunction with the patient's primary needs is a productive assessment method with several positive outcomes.

Objective: The article aims to present the importance of understanding the relationship between pain and psychological distress-related symptoms and how it influences pain intensity. The study also explores the existing psychological methods and techniques of pain assessment for quality of life.
\end{abstract}

Material and method: The present study is a qualitative retrospective method on existing literature and documents.

Key words: pain, psychological symptoms, cognitive-behavioral therapy, anxiety, opioid

\section{Introduction}

"We must all die. But that I can save him from days of torture that is what I feel as my great and ever new privilege. Pain is a more terrible lord of mankind than even death itself." - J.J. Bonica.

Being with pain is a stressful, complicated, and multifactorial experience that destroys individual cognitive and behavioral functioning in dealing with pain symptoms. These psycho-emotional feelings depict the level of pain that maximizes the level of anxiety, stress, and depression. Pain is the one factor that proved the direct link between the human mind and body. The perception of pain demands different understanding paradigms in defining and seeking multidimensional approaches through multidisciplinary team interventions in clinical practices. Pain is not confined within the bio-medicinal realm alone; it is a wide-ranging phenomenon that requires holistic assessment. There are multiple layers of complex thoughts and feelings in the human brain that construct the level or the amount of pain a person experience and can tolerate. Since it is the human thoughts and feelings that determine how the individual deals with pain symptoms, controlling personal perception and emotion can serve as an effective coping mechanism to modify and cope with the physical pain symptoms [1]. Thus, understanding the psychological aspect of human pain and symptom could significantly enhance patient treatment and deliver the quality of life. The psychological assessment is visible, playing an essential role in managing and reducing patient discomfort and other pain-associated problems. 


\section{Psychological distress and physical pain symptom}

Pain is the factor that ruins everyday behavior and interrupts all activities, and is more than a sensational experience. It is a complex component of both physiological and psychological responses in the individual experience. Pain is an unpleasant combination of human sensory and emotion that damages tissues, brain, and memory functioning. The pain symptom demands regular comprehensive assessment to identify the patient physical, psychological, social, and spiritual dimensions through multidisciplinary team interventions for psychodynamic and neurotic assessments. The perception of pain is an individual response to factors like childhood experience, socio-cultural, heredity that usually produces different aspects of pain and suffering. Pain becomes more severe and unbearable, mainly to those with a distressing socio-cultural group of people. In contrast, the pain thresholds were at a lower rate among the socioculturally stable group of people with faster recovery rates. The context of pain and the behavioral condition of the patient also hugely determine the severity perception of pain experienced by the individual, which requires cognitive and practical aspects of psychological care assessment to the earliest [2]. In all the existing ill-related experiences, pain is the underlying source that produces disability and risk anxiety disorders leading to several suicidal activities. Several studies recognize that pain intensity in its nature of existence is in connection with psychological symptoms. The longer duration of pain, the higher intensity of mood disorder, depression, and anxiety increase the level of pain symptoms. The fMRI (Functional Magnetic Resonance Imaging) mainly depicts how pain symptoms influence mood disorders and emotional aspects in clinical practices. Most research findings stated that the psychological depressive symptoms and pain frequency are the two determining factors of human health. The epidemiological evidence also affirms the high intensity of psychological distress over pain symptoms among medically advance ill patients. The ovarian hormones are also equally responsible for pain intensity in a different region of the human brain/mind state [3].

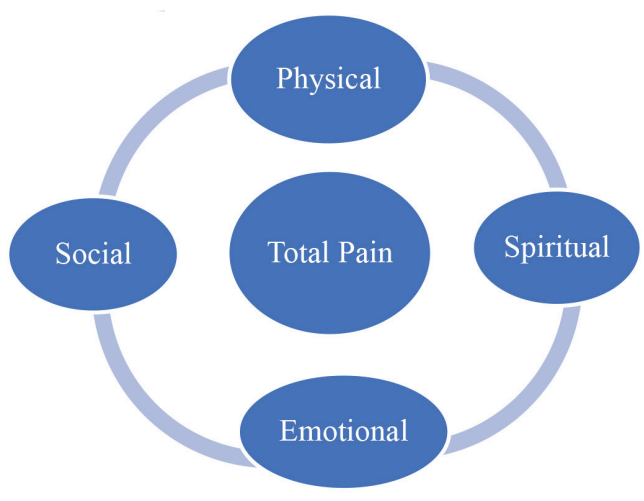

Thus, understanding the individual concept of physical pain is essential to understand the endurable pain level and develop effective coping strategies in dealing with both acute and chronic pain symptoms. Chronic pain accompanied several psychological and mood disorders that usually destroyed patients' peace of mind and quality of life in their nature of existence. The factors responsible for the causes of chronic pain in the human body are; nerve fibers dysfunction through injuries or surgery, patient medical condition, some portions of brain neurons dysfunction, through accident or illness [4].
Another finding suggested that the particular immediate context of occurrences hugely influences intensity over acute pain syndrome. Thereby, if the individual is preoccupied with existential fear and anxiety, even opioids and other related drugs are visible less effective. It instead worsens the intensity of physical pain syndrome. The recent experimental study also found the feeling of hypochondria or somatic visible in increasing the intensity of physical pain symptoms. On the other hand, timely addressing the pain syndrome by distracting the patient thoughts into another interest is an effective coping mechanism that reduces the sensitivity rates of pain and suffering [1]. Pain is a distressful and prominent symptom that affected $75-78 \%$ of patients with advanced medical illness. It is also evidence that $68 \%$ of death due to stroke have experience pain and symptom disorders. The study also depicted that pain syndromes highly affected the individual ways of life, physical functioning, concentration, increasing disease risks, and helplessness. Being with pain is a stressful event that makes the psychological factors central to the individual experience of pain and for quality treatment policy. Stress is the contributing factor for health problems and chronic pain, including blood pressure, heart disease, obesity, diabetes, and trigger muscle tension that maximize pain intensity. The treatment procedure demands the associations of the sensory, cognitive, emotional, behavioral, and environmental factors that need immediate assessment in the possible ways for effective pain management [5].

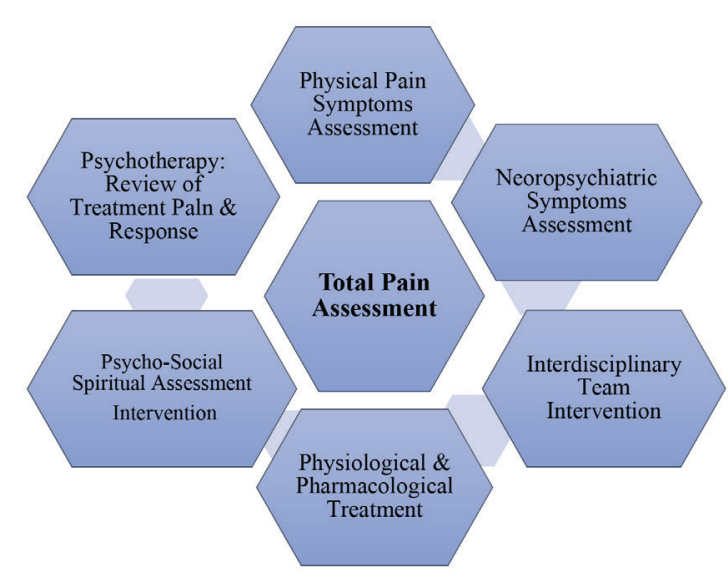

Adapted from Bettina Sandgathe Husebø, 2020[7].

\section{The Psychological Aspects of Pain and Symptom Management}

Despite the advanced modern medical science and technologies in clinical practices, psychological distress remains the underlying issue in dealing with chronic pain. The intensity of psycho-emotional grief associated with pain increases disease risk rates in the individual experience and reduces inpatient quality of life. Pain in its nature of existence is a complex psychoneurotic syndrome that requires a multidisciplinary inclusive approach to deal with complicated disorders. Effective total pain management hugely depends on the holistic assessment of the patient's physical, psycho-emotional, social, and spiritual aspects. Identifying psychological distress-related symptoms and the significant examination of factors associated with painrelated distress are the two essential elements to be identified in pain assessment. Identifying the patient's pain frequency and the amount of pain accounted for health variables is also equally crucial as it significantly differs from the individual cognitive-biological and environmental context [6]. Thus, the 
psychotherapeutic interventions inpatient total pain management becomes an essential coping mechanism in reducing the intensity of pain. The psychological assessment is also visible in identifying and understanding the patient's negative thoughts, emotional sufferings, and other associated ill behavior, which usually produces discomfort and increases the pain inpatient experience. The pain syndrome needs proper assessment by understanding specific components associated with pain and through the optimal comprehensive assessment interventions at a multidisciplinary level. The psychopharmacology aspect of pain management in conjunction with the patient's primary needs is a productive assessment method with several positive outcomes [5].

\section{Adapted from Bettina Sandgathe Husebø, 2020[7].}

On the other hand, recognizing the role of psychologists in pain management begins during the 1950s and 1960s with the rapidly growing problems of patients experiencing disabilities through constant chronic pain. On the other hand, the patients were not able to explain the extent of damage caused in their daily experience with the pain symptoms. In finding the nature of pain that interrupts the individual cognitive function, the clinicians focus on biomedicine asper and ignore the psychological factors accompanying the pain syndrome. Failing to acknowledge the psychological components resulted in long-term psychological damage and makes the pharmacological treatment ineffective [8]. In addressing the total pain, it is essential to understand the context and the factors involved. The cognitive-behavioral psychotherapeutic is the one approach that effectively addresses the nature of real pain in the context where it occurs and the associated factors. Cognitive Behavior Therapy is a humanistic approach that constructs unique coping skills through several relaxation techniques for managing and controlling the total pain symptoms. It adopts the diversion techniques against the psychosocial-spiritual signs that accompanied the patient physical pain through its positive therapeutic approach. The therapeutic assessment effectively manages specific body functioning, brain waves, stress response, relaxing muscular tension, and reducing the overall body response to pressure [9]. Acknowledging the patient's socio-cultural background, economic status, and belief system is another critical area to be identified to deliver quality treatment procedures in total pain management.

The biopsychosocial model is a modern humanistic approach that incorporates patient sensory, cognitive, emotional, behavioral, and environmental factors in understanding and managing the pain symptoms. Mainly for adults with chronic pain, the cognitive-behavioral model is an essential coping mechanism for relieving pain and recover from symptoms. The study on pain experience among the medically ill population found that pain is due to patient psycho-emotional and behavior factors like beliefs, fear, and addiction to drugs. In addition to chronic pain, the majority of the patients experience physical organ dysfunction, sleep disorder, fatigue, isolation, and care assessment dissatisfaction. The side effects of pain hugely affect the patient's cognitive process, emotional outbreak, and other phobic responses. It requires the psychological understanding and assessment of patient pain experience alongside the biomedicinal supports for effective pain management in clinical practices. On the other hand, the psychosocial approach is the model of care that encounters the environmental challenges and other inevitable cognitive issues in the chronic pain experience.
A pilot study done on chronic pain management shows the increasing reliability of the psychological instruments used to treat patient cognitive-behavioral therapy towards chronic pain. The treatment aims to reduce the patient's maladaptive behavior, beliefs, negative thoughts and identify those associated harmful components that make pain treatment ineffective. The therapeutic assessment helps in re-organizing the patient cognitive process through pain therapists, anesthetists, clinical psychologists, and physiotherapists in the clinical practices. The pain management medicinal contents differ as per the patient's perception of the pain and cognitive-behavioral context. It is basing on seven fundamental principles; Direct positive reinforcement of pain behavior, Indirect positive support of pain behavior, Positive reinforcement of good behavior, Physical fitness and function, Cognitive reframing, Education and empowerment, Critical process factors, and The evidence base $[10,7,8]$.

\section{Challenges and discussion}

The emerging issues in pain and symptom management are opioids and other related drugs that lead to addiction or total dependency. This addiction and total dependency make the psychological intervention more essential to control the dependence or total reliance on opioids and other drugs through its non-drugs psychotherapeutic techniques. The patient mainly develops total dependence on drugs in chronic pain when their psychosocial-emotional sufferings were not identified and treated on time. On the other hand, chronic pain is a stressful event, subjective experience, self-limiting but not self-evident, which becomes the underlying public health concern. To encounter frustrating patient events and difficult situations effectively demands multimodal approaches to care with multidisciplinary team interventions. No doubt that humans are destined to die, but the pain is a terrible lord rather than death itself. So, saving the individual from the torture of pain and its side effects becomes the core concern for the multidisciplinary teams involves in clinical pain management [11]. However, in a country like India, the distressing psychological symptoms are still considered as symptoms not to be treated in its clinical practices. The painful patient symptoms and emotional suffering are not considered an illness to be treated in most cases. It is only through identifying and acknowledging the psychosocialspiritual distressing symptoms associated with pain, the influential 'total pain' management policy can be successfully implemented. The holistic assessment in clinical practices is satisfactory for the patient and is a rewarding activity even for the health care professionals.

The war against chronic pain symptoms remains a difficult one in clinical practices. It is always co-existed with several psychological distressing symptoms, which sometimes resulted in a patient physical disability. Albert Schweitzer wrote: "The use of narcotics in terminal cancer is to be condemned if it can be avoided. Morphine and terminal cancer are in no way synonymous. Morphine usage is an unpleasant experience for the majority of human subjects because of its undesirable side effects. Dominant in the list of these unfortunate effects is addiction" [12]. The psychological non-drugs therapeutic approach is the only way to relieve pain that prolongs suffering without addiction and zeroes adverse side effects. Pain management is a unique challenge to health care professionals that requires several sensible and pragmatic diagnostic approaches to intervention, alongside quality management skills and strategies. At present, realistic expectations and recognition of the limitation in chronic pain medication are the two emerging challenges 
in chronic pain management. The use of an opioid is another fundamental challenge to every health care professional. The dangers of opioids and other related drugs can be mainly visible in worsening a patient's ill condition, loss of memory, untimely death, and maximizing tension if overprescribed. It requires an appropriate pain management policy and comprehensive strategies that work in the patient's best interest with the minimal inclusion of the use of opioids or total avoidance of opioids and other related drugs. The pain management strategies should optimize effectiveness with minimal adverse side effects through the psychological mind-body therapeutic approaches. The primary aim of chronic pain management is not to eradicate the pain, instead to help the patient adjust its thresholds through appropriate care interventions [13].

In chronic pain management, the patient and even those clinicians addressing the pain symptoms experience several psychological disorders like stress, stigmatizes, isolation, and being disturbed in their everyday life that require special considerations. Thus, psychological issues become the central concern in dealing with pain symptom management in clinical practices. It is to be noted that improving the pain management standards will inevitably enhance the patient/clinician's quality of life and wellbeing of the whole in any clinical practice. The psychological cognitive-behavioral therapeutic approach is the existing effective model of care to encounter the complexity of pain experience through its biofeedback and relaxation therapy. It is essential to establish a patient referral model to pain clinics, financial support, acknowledging patient attribution, and consistent evaluation of patient treatment outcome or progress in pain management. Patient physical fitness also plays a vital role in managing pain-related symptoms that can provide patient self-reinforcement with a positive outlook towards environmental challenges. Some of the underlying factors that need to be evaluated in psychological pain assessment are; care goals, present/past adherence to diagnosis/prognosis, patient beliefs, and cultural background. Comorbidities and related symptoms apart from the pain syndrome, patient history of drugs consumptions, ongoing patient cognitive functioning with the treatment, and socio-economic status are also essential [5].

The clinicians should be cautious in differentiating between the patient's physiological tolerance, physical and psychological dependency on opioids, and related drugs in pain assessment. The amount of medications for pain control and the negative phenomenon after the withdrawal of drugs is an important domain that requires special considerations. The psychological assessment is another critical area of care to help the patient develop the ability to create self-defeating and self-denigrating insights to encounter reality and pseudo-addiction behaviorism. It will also help enhance the individual capability of stress management to undergo effective self-relaxation therapy that will lead the individual to identify and solve their problems. The psychological assessment is also visible effective in reforming or improving the daily habits of the patient physical activity by not allowing pain symptoms to take over the individual happiness. Developing a healthy lifestyle, distracting pain through social integration, and engaging with newly form hobbies is also an essential coping mechanism against pain syndrome [14,7]. The psychological aspect of pain management is a growing public health concern that identifies the patient pain frequency to establish a quality treatment plan and policies. The psychological cognitive-behavior and evidence-based strategical therapeutic approaches are effective with chronic pain of every age and complex cultural background. The psychospiritual model is also another effective pain and symptom management mechanism, mainly with those psychological depressive symptoms through chronic pain [15]. Thus the underlying factor in pain management is to acknowledge both the mental and physical risk factors for effective pain management strategies. The concept of total care approaches that address patient socio-economic and other bio-environmental challenges through multidisciplinary team interventions could effectively help the patient in their pain experience than any other method.

\section{Conclusion}

Pain is a stressful, complicated, and multifactorial experience. It is a universal symptom that disturbs the whole process of normal human organ functioning. Whether acute or chronic pain it is always accompanied by several unwanted, ill feelings that increase the disease risk. The psychological assessment is a safer approach of care that provides a non-drug method that directly deals with the origin of pain symptoms. It helps reduce the psychological depressive stressors that worsen the individual pain and cope effectively with several factors associated with pain and suffering. Thus, learning how to cope with the problems and stressors related to pain and suffering is the core criterion for effectively dealing with the pain symptoms.

Disclosures: There is no conflict of interest for all authors.

\section{Acknowledgements: None.}

\section{Funding: None.}

Informed consent: Informed consent was obtained from all individual adult participants included in the study; assent was obtained from children.

\section{References}

1. George R. Hansen, George R \& Streltzer, John. The Psychology of Pain. Emerg Med Clin N Am. $2005 ; 23$ (1): 339-348. Doi:10.1016/j.emc.2004.12.005.

2. Meldrum ML. Pain: Physiology and Psychology in Encyclopedia Britannica. https://www.britannica.com/science/pain. Accessed from the web on December 17, 2019.

3. Gorczyca R, Filip R, Walczak E. Psychological Aspects of Pain. Ann Agric Environ Med. 2013; Special Issue (1):23-27.

4. Intermountain Healthcare. Pain and Symptom Management. https://intermountainhealthcare. org /services/hospice-palliativecare/services/pain-and-symptom-management. Accessed on December 18, 2019.

5. Rizk Dahlia. Palliative Care: Pain Management. https://www.cancertherapyadvisor. com/home/decision-support-in-medicine/ hospital-medicine/palliative-care-pain-management/. Accessed on December 18, 2019.

6. Baker, T.A., Krok-Schoen, J.L. \& McMillan, S.C. Identifying factors of psychological distress on the experience of pain and symptom management among cancer patients. BMC Psychol. 2016; 4 (52): 1-7. Doi:10.1186/s40359-016-0160-1. 
7. Sandgathe Husebø et al. Innovating the clinical pathway for home-dwelling people with dementia and their caregivers: study protocol for a mixed-method, stepped-wedge, randomized controlled trial. Trials. 2020; 21(510). Accessed from the web https:// trialsjournal.biomedcentral.com/articles/10.1186/s13063-020-04414-y.

8. Eccleston C. Role of psychology in pain management. BJA: British Journal of Anaesthesia. 2001; 87 (1); 1:144-152, https:// doi.org/10.1093/bja/87.1.144.

9. APA. Managing Chronic Pain: How psychologists can help with pain. managementhttps://www.apa.org/helpcenter/painmanagement. Accessed on December 19, 2019.

10. Zara, C, Baine, N. Cancer pain and psychosocial factors: a critical review of the literature. Journal of Pain and Symptom Management. 2002;24:526-542. https://doi.org/10.1016/S0885-3924(02)00497-9

11. Turk DC, Rudy TE. Neglected factors in chronic pain treatment outcome studies - referral patterns, failure to enter treatment, and attrition. Journal of Pain. 1990; 43(1): 7-25. DOI: 10.1016/0304-3959(90)90046-g.

12. Bonica J. J. History of pain concepts and therapies. 1990: 2-13. In Thomas MA. Pain management - the challenge. Ochsner Journal. 2003; 5(2):15-21.

13. Thomas MA. Pain Management - The Challenge. Ochsner Journal. 2003; 5 (2):15-21.

14. Harvard Medical School. Challenges of Managing Chronic Pain. https://www.bmj.com/content/356/bmj.j741. Accessed on December 22, 2019.

15. Groninger Hunter. Pharmacologic Management of Pain at the End of Life. American Family of Physician. $2014 ; 90(1): 27-32$.

16. Lee, Y., Wu, C., Chiu, T. et al. The relationship between pain management and psychospiritual distress in patients with advanced cancer following admission to a palliative care unit. BMC Palliat Care. 14(1):1-7. Doi:10.1186/s12904-015-0067-2. 